\title{
Patient Satisfaction Index and Quality of Life Measurement with Breast- $Q$ after Breast Reconstruction in a Plastic Surgery Center in Mexico
}

\author{
Jose C. Martinez-López, Jaime A. García-Espinoza, David Flores-Soto, \\ Carlos I. Navarro-Delgadillo, Cuahutemoc Márquez-Espriella, Rodrigo Dávila-Diaz, \\ Esteban I. Campos-Serna, Víctor H. Avalos-Gómez
}

Division of Plastic and Reconstructive Surgery, Hospital Central Sur de Alta Especialidad de Petróleos Mexicanos, Mexico City, Mexico

Email: jocar.ml@hotmail.com

How to cite this paper: Martinez-López, J.C., García-Espinoza, J.A., Flores-Soto, D., Navarro-Delgadillo, C.I., Márquez-Espriella, C., Dávila-Diaz, R., Campos-Serna, E.I. and Avalos-Gómez, V.H. (2021) Patient Satisfaction Index and Quality of Life Measurement with Breast-Q after Breast Reconstruction in a Plastic Surgery Center in Mexico. Journal of Biosciences and Medicines, 9, 94-105.

https://doi.org/10.4236/jbm.2021.96009

Received: May 2, 2021

Accepted: June 15, 2021

Published: June 18, 2021

Copyright $\odot 2021$ by author(s) and Scientific Research Publishing Inc. This work is licensed under the Creative Commons Attribution International License (CC BY 4.0).

http://creativecommons.org/licenses/by/4.0/ (c) (i) Open Access

\begin{abstract}
Introduction: Cancer is one of the most devastating pathologies to affect the breast. Mastectomy stigmas are associated with depression, body image dysmorphia, and decreasing quality of life. BREAST-Q is a PROM (Patient Reported Outcome Measures) that has proven useful in measuring satisfaction with breast reconstruction results from the patient's point of view. Objectives: To measure the satisfaction index and improvement in quality of life after breast reconstruction for breast cancer sequelae in our hospital in the last 5 years. Materials and Methods: Descriptive Study that includes patients with Breast cancer diagnosis that underwent mastectomy and breast reconstruction (prosthesis or autologous tissue) in "Hospital Central Sur de Petroleos Mexicanos" (January 2015 to January 2020), whose satisfaction index was measured with BREAST-Q one year after reconstruction. Results: 153 patients were included in the analysis. Mean global satisfaction was 74 points. We observed a tendency towards higher psychosocial, sexual and appearance satisfaction in patients who underwent reconstruction with autologous tissue. The mean satisfaction with provided information was 64 points and with the medical team $>90$ points. Conclusion: Breast reconstruction is associated to a high satisfaction index and quality of life improvement regardless of the technique. BREAST-Q proved to be useful in evaluating patient experience and it helped us identify areas of opportunity to improve our care.
\end{abstract}

\section{Keywords}

BREAST-Q, Breast Reconstruction, PROM's, Satisfaction Index, 
Quality of Life, Mexico

\section{Introduction}

Cancer is one of the most devastating pathologies to affect the breast, the organ that represents the center of femininity and a unique body characteristic in the female body. In 2018 over 450 thousand cases of breast cancer were diagnosed in America, and it was responsible of over 100 thousand deaths [1]. In Mexico it accounts for $15 \%$ of new cancer diagnoses and has a mortality rate of 17.2 deaths for every 100 thousand women. The latter demonstrates that it is a highly frequent disease with high morbidity and mortality, making it a national health problem in need of priority attention.

Currently, a mastectomy is considered the treatment of choice for early stages, nevertheless it has been proven that its disfiguring stigma is related to depression, body image dysmorphia and decreasing quality of life [2] [3]. With this problem in mind, multiple surgical techniques have been developed, and are gathered in an article by Pinell and Giroux [4].

To evaluate postoperative satisfaction from the patient's point of view, early studies with non-reproducible tools showed an improvement in quality of life and self-perception, even if the reconstruction process was not completed (nipple areola complex reconstruction) [5] [6]. This constituted one of the reasons why breast reconstruction is now an integral part of breast cancer treatment in multiple nations, including Mexico.

Due to the need for measuring treatment impact on the patient in an objective, reproducible and standardized manner, during the 1950's PROM's began development in Europe [7]. In 2009 Pusic et al. published BREAST-Q, which considers quality of life and satisfaction after breast reconstruction [8]. Currently BREAST-Q has been validated in over 30 languages including Spanish and has proven useful to contrast the impact on quality of life between different surgical techniques [9], and to encourage changes in health care policies based on scientific evidence [10]. Despite its multiple applications, a systematic review of the literature in 2015 reported only 39 studies that mentioned the Reconstruction module of BREAST-Q [9], and only a few of these were published in Latin America.

In these studies, expectation and cosmesis perception from the patient's patient point of view played a key role in determining satisfaction and quality of life after reconstruction [11] [12] [13]. A more recent study published in Saudi Arabia concluded that the systematic application of BREAST-Q allows for a more integral treatment, offering surgical options that achieve a higher impact on self-perception, sexual life, self-esteem, and quality of life [14].

After researching national and international indexed databases using key words such as: "breast reconstruction", "satisfaction index", and "Mexico", no 
studies were found that use standardized tools to measure satisfaction after breast reconstruction in the Mexican population. For this reason, we decided to measure satisfaction index and quality of life improvement after unilateral and bilateral breast reconstruction for breast cancer sequelae in our institution, with the goal of gathering objective information that we can share with patients that are about to begin the process of reconstruction, as well as to compare results from the reconstructive techniques that we use most often, and therefore determine which is the best procedure for our patients.

\section{Materials and Methods}

It is a retrospective study including patients with breast cancer diagnosis, who have undergone breast reconstruction surgery using any technique (prosthesis and autologous tissue) in "Hospital Central Sur de Alta Especialidad de Petroleos Mexicanos" from January 2015 to January 2020. Records must include BREAST-Q Reconstruction module questionnaire completed one year after reconstruction.

We used the BREAST-Q (Licensed Version 2.0 Reconstruction Module) in the Spanish Version, published by The Memorial Sloan Kettering Cancer Center and The University of British Columbia. This questionnaire contains 111 items divided in 16 modules that evaluate: Psychosocial well-being, sexual well-being, Physical well-being regarding the chest and abdominal areas, satisfaction with breasts, implants, abdomen and nipple reconstruction; adverse effects of radiation and patient experience regarding satisfaction with information, surgeon, medial team and office staff. A copy of the BREAST-Q in the English version can be found in the qportfolio website:

https://qportfolio.org/breast-q/breast-cancer/.

The sum score of the answers were transformed into a score from 0 (worst) to 100 (best) using the Equivalent RASCH tables provided for each module in the BREAST-Q. This way satisfaction was stablished with higher scores reflecting a better outcome. All questionnaires were filled by the patients themselves under medical supervision and scored by two of the authors (JCML \& DFS).

Electronic records were reviewed, and the following epidemiological variables were recorded: age, BMI (Body Mass Index), affected side, histologic cancer diagnosis, postoperative complications, chemotherapy and radiotherapy treatment, time from mastectomy to beginning of reconstruction process, time to complete breast reconstruction (nipple areola complex reconstruction), or whether the patient no longer desired to undergo more procedures.

All patients who underwent breast reconstruction for diagnoses other than breast cancer, those who did not have a complete medical record including the variables, or those who did not answer the questionnaire were excluded from the study. Study groups were divided according to the type of reconstruction technique they underwent: "Direct implant”, "Expander-Implant”, “TRAM flap" and "Latissimus Dorsi flap + Implant". A general description was carried out for 
each of the BREAST-Q 2.0 questionnaire variables, and the scores obtained for each of the treatment groups were compared.

For the statistical analysis, a non-parametric analysis with central tendency measures (mean, median and mode) was performed, as well as dispersion measurements (standard deviation and interquartile range) using Excel 2010.

This protocol was previously reviewed and approved by the Research and Ethics Committee of our Institution. All procedures were in line with the regulations of the General Health Law in matters of health research in Mexico.

\section{Results}

Between January 2015 and January 2020, a total of 153 patients with sequelae of mastectomy due to breast cancer underwent breast reconstruction in our hospital. All of them had a complete medical record and completed the BREAST-Q a year after undergoing reconstruction.

The median age of the group was 56 years (SD 14.2), the mean weight was $68.75 \mathrm{~kg}$ (SD 8.41), the mean BMI was $29.2 \mathrm{~kg} / \mathrm{m}^{2}$ (SD 3.81), the median time between mastectomy and reconstruction in months was 30 months (SD 17.5), the median duration of the reconstruction process in months was 12.5 months (SD 5.3), and the incidence of complications was 13\% (Table 1).

From the 153 patients, 66 were reconstructed with autologous tissue, of which 22 (14.37\%) underwent "Latissimus dorsi flap + implant", and 42 (27.4\%) underwent "TRAM flap"; 89 patients were reconstructed with prosthesis, of which 69 (45\%) underwent "Expander-Implant" and 20 (13\%) underwent direct implant (Table 1). The patients were therefore divided into four groups, depending on the type of reconstruction they underwent.

Latissimus dorsi flap + implant: for this group the median age was 45 years (SD 3.6), the mean weight was $74 \mathrm{~kg}$ (SD 9.6), the mean BMI was $30.7 \mathrm{~kg} / \mathrm{m} 2$ (SD 4.8 ), the median time between mastectomy and reconstruction in months was 22.6 months (SD 10.5), the median duration of the reconstruction process in months was 24.3 months (SD 5.3), and the incidence of complications was $10.9 \%$, with the most frequent being wound dehiscence (Table 1).

TRAM flap: the median age of the group was 53.2 years (SD 6.1), the mean weight was $67.5 \mathrm{~kg}$ (SD 7.2), the mean BMI was $26.6 \mathrm{~kg} / \mathrm{m} 2$ (SD 3.01), the median time between mastectomy and reconstruction in months was 48.6 months (SD 20.1), the median duration of the reconstruction process in months was 18.7 months (SD 10.1), and the incidence of complications was $21.1 \%$, with the most frequent being partial flap necrosis (Table 1).

Expander-Implant: the median age of the group was 52.3 years (SD 15.1), the mean weight was $66.1 \mathrm{~kg}$ (SD 22.4), the mean BMI was $25.96 \mathrm{~kg} / \mathrm{m} 2$ (SD 3.71), the median time between mastectomy and reconstruction in months was7.6 months (SD 6.9), the median duration of the reconstruction process in months was 12.08 months (SD 6.5), and the incidence of complications was 3.4\%, with the most frequent being expander exposure and partial necrosis of the nipple 
Table 1. Demographic characteristics of patients who underwent breast reconstruction in our institution from January 2005 to January 2020 ( $n=153)$, divided by procedure. Data presented in the table is expressed as mean with standard deviation.

\begin{tabular}{cccccc}
\hline Demographic Characteristics & Global & $\begin{array}{c}\text { Latissimus Dorsi } \\
\text { + Implant }\end{array}$ & TRAM Flap & $\begin{array}{c}\text { Expander } \\
\text { + Implant }\end{array}$ Direct Implant \\
Total & $153(100 \%)$ & $22(14.4 \%)$ & $42(27.4 \%)$ & $69(45 \%)$ & $20(13 \%)$ \\
Age (years) & $56(14.2)$ & $45(3.6)$ & $53.2(6.1)$ & $52.3(15.1)$ & $56(4.2)$ \\
Weight (Kg) & $68.8(8.4)$ & $74(9.6)$ & $67.5(7.2)$ & $66.1(22.4)$ & $68.7(8.4)$ \\
Height (m) & $1.5(0.06)$ & $1.6(0.02)$ & $1.6(0.04)$ & $1.6(0.07)$ & $1.5(0.09)$ \\
BMI (Kg/m2) & $29.2(3.8)$ & $30.7(4.8)$ & $26.6(3.01)$ & $26(3.71)$ & $29.2(7.1)$ \\
Time from mastectomy to reconstruction (months) & $30(17.5)$ & $22.6(10.5)$ & $48.6(20.1)$ & $7.6(6.9)$ & $30(26.9)$ \\
Duration of reconstruction process (months) & $12.5(5.3)$ & $24.3(9.3)$ & $18.7(10.1)$ & $12.1(6.5)$ & $12.8(6.1)$ \\
Complicaciones (\%) & $13 \%$ & $10.9 \%$ & $21.1 \%$ & $3.4 \%$ & $6.9 \%$ \\
\hline
\end{tabular}

areola complex (Table 1).

Direct implant: the median age of the group was 56 years (SD 4.2), the mean weight was $68.7 \mathrm{~kg}$ (SD 8.4), the mean BMI was $29.2 \mathrm{~kg} / \mathrm{m}^{2}$ (SD 7.1), the median time between mastectomy and reconstruction in months was 30 months (SD 26.9), the median duration of the reconstruction process in months was 12.7 months (SD 6.1), and the incidence of complications was 6.9\%, with the most frequent being implant exposure and abnormal scar (Table 1).

In the psychosocial wellbeing module, we observed an overall wellbeing status of $>74$ points, regardless of the type of reconstruction, with a tendency towards higher perception of wellbeing in patients who underwent autologous tissue reconstruction (93 and 87 vs. 83 and 77 points) (Graphic 1).

In the Sexual wellbeing module, we observed an overall wellbeing status of $>62 \%$ regardless of the type of reconstruction, with a tendency towards a higher perception of wellbeing in patients who underwent reconstruction with TRAM flap (74 points), and worse perception in those who underwent direct implant reconstruction, scoring an average of 12 points below the global average (Graphic 1).

In the chest appearance satisfaction module, we observed a median satisfaction of $71 \%$ regardless of the breast reconstruction technique. In this case, patients who underwent reconstruction with latissimus dorsi flap + implant reported $100 \%$ satisfaction with their results (Graphic 2).

In the appearance satisfaction between the two breasts module, we observed a global satisfaction of $67 \%$. Once again, patients reconstructed with Latissimus dorsi flap + implant reported to be the most satisfied with their appearance (Graphic 2).

Among the patients whose reconstruction involved the use of an implant, we observed a tendency towards being more satisfied with the involvement of autologous tissue techniques (100 vs. 92 and 81) (Graphic 2).

In the Abdominal appearance satisfaction module, we found a clear tendency towards higher satisfaction in patients who underwent TRAM flap (89.3) versus 


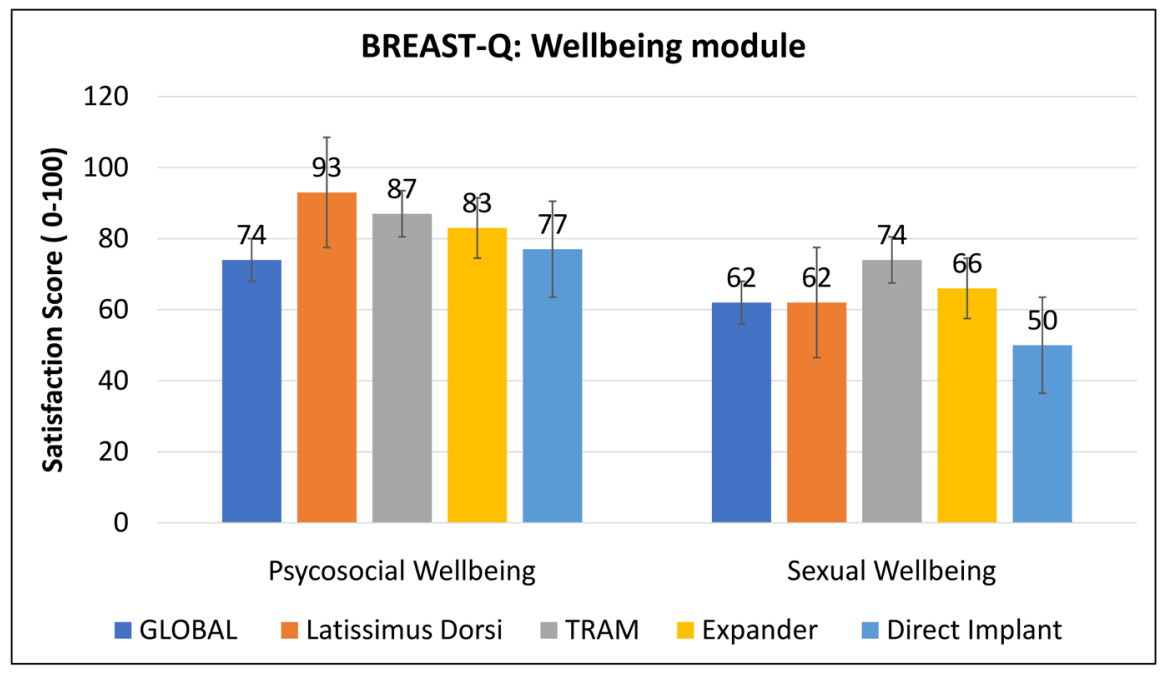

Graphic 1. Satisfaction scores (+SD) of each group in the Psychosocial and sexual wellbeing modules. Global $(n=153)$, Latissimus Dorsi $(n=22)$, TRAM $(\mathrm{N}=42)$, Expander $(\mathrm{n}=69)$, Direct implant $(\mathrm{n}=20)$.

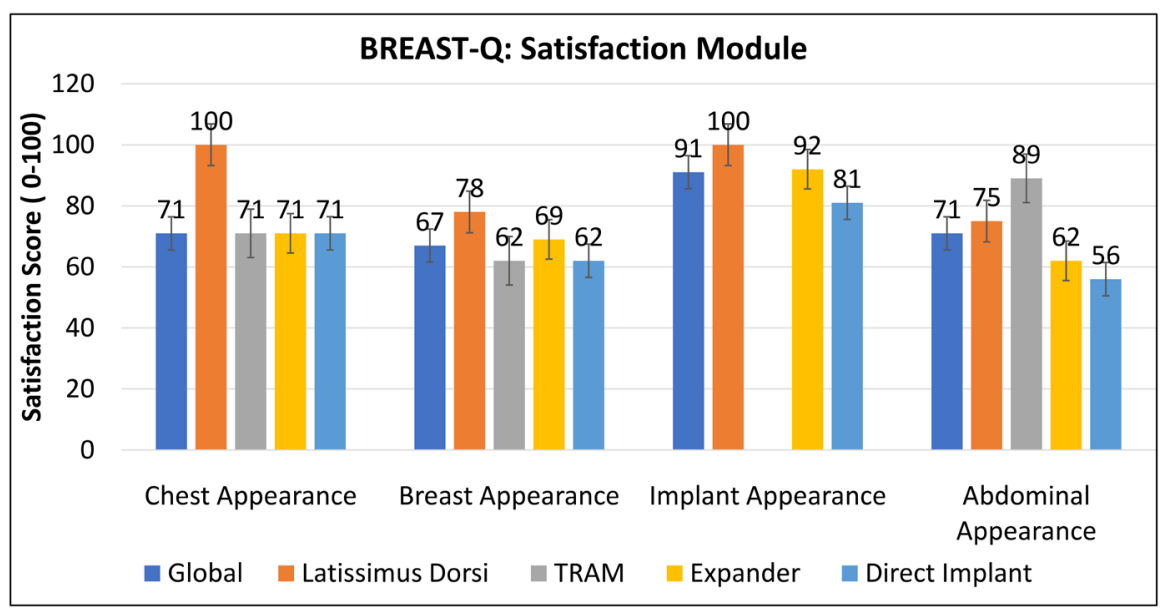

Graphic 2. Satisfaction scores (+SD) of each group in the Chest, Breast, Implant and Abdominal Appearance modules. TRAM Scores are not included in the Implant Appearance module, since none of the procedures in this group included the placement of an implant in our hospital.

implant-based techniques (62 and 56.3), without evidence of higher incidence of abdominal symptoms (Graphic 2).

In the Physical wellbeing module regarding the chest and abdominal area, a higher incidence of symptoms was reported in patients who underwent direct implant reconstruction, associating it with less satisfaction. The least number of symptoms and highest satisfaction index were reported in patients reconstructed with expander-implant and Latissimus dorsi respectively (Graphic 3).

The percentage of patients that received radiotherapy was higher in patients reconstructed with Latissimus dorsi (33\%), compared with the average of the other groups (<25\%) (Graphic 4).

The satisfaction score regarding Radiotherapy Sequelae was higher in patients 


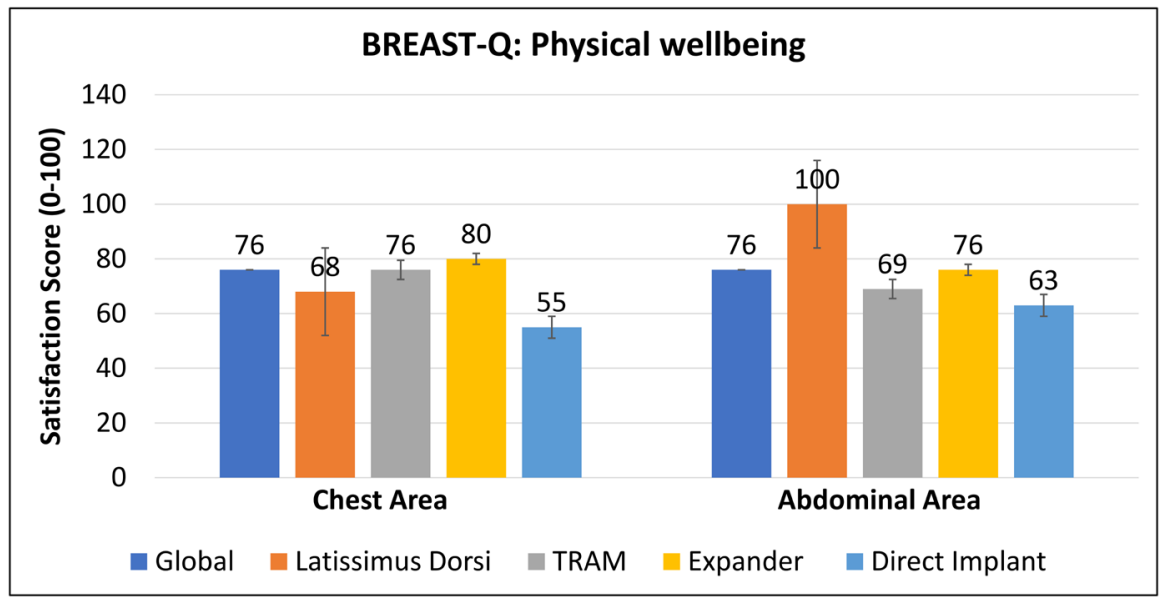

Graphic 3. Satisfaction scores (+SD) of each group in the physical wellbeing modules regarding symptoms in the Chest and Abdominal Areas.

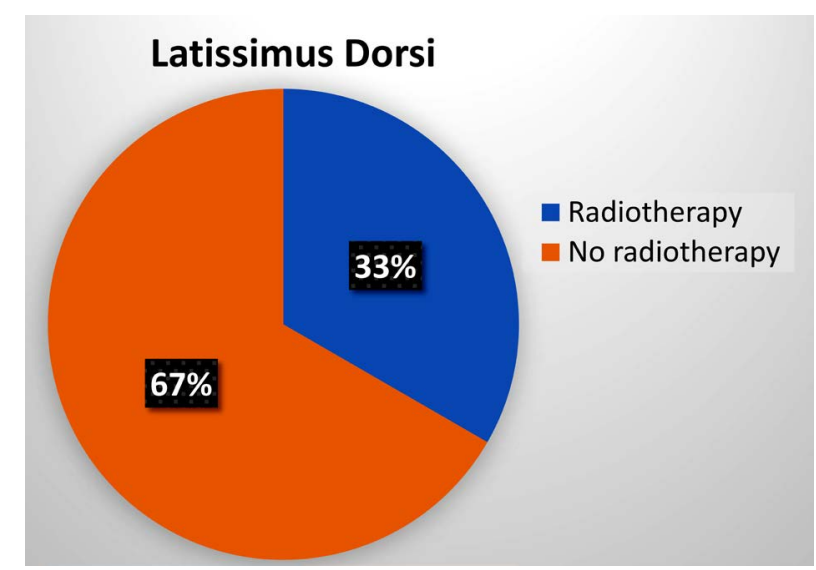

Graphic 4. Percentage of patients (\%) that received radiotherapy in the group reconstructed with Latissimus dorsi $(n=22)$.

reconstructed with autologous tissue-based techniques ( $>78$ points) versus Expander (71 points), and it was more evident when compared with Latissimus dorsi group alone (87 points) (Graphic 5).

Regarding satisfaction with the information given to the patient during the reconstruction process the mean score was 64 points (Graphic 6).

In the modules that evaluated patient satisfaction with medical care, all the groups reported a high satisfaction index, without an evident difference amongst them (Graphic 7).

\section{Discussion}

Breast cancer is the most common cancer and the second cause of death by cancer amongst women in America [1]. The economic situation of third world countries is particularly associated with greater consequences of this illness. One of the tasks of the plastic surgeon is to mitigate these consequences and try to restore what cancer took away from them. For more than 3 decades, we have known that there is an improvement in quality of life and patient satisfaction 


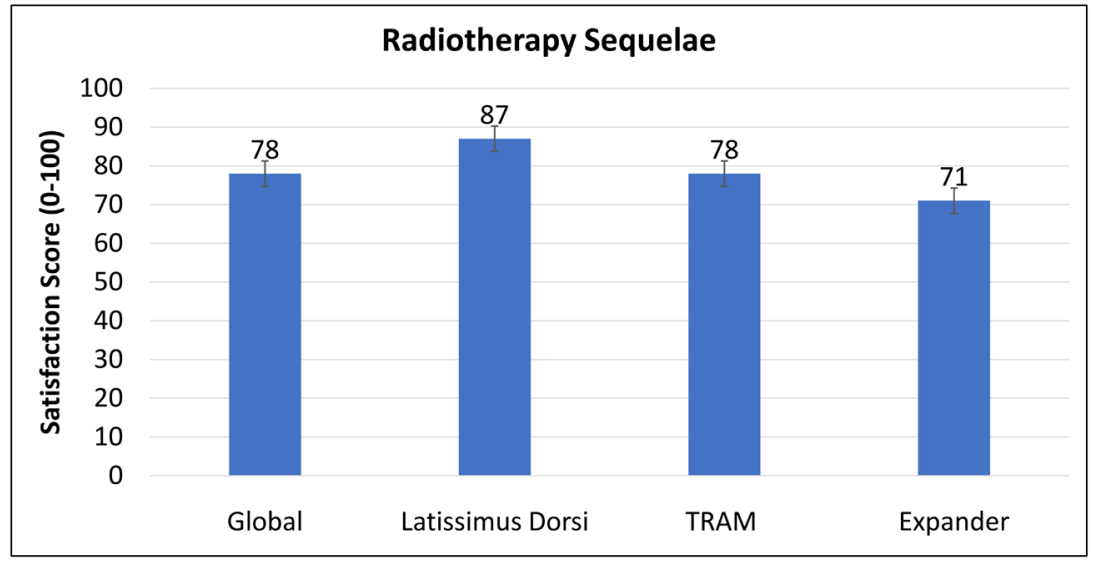

Graphic 5. Satisfaction Score (+SD) of Radiotherapy Sequelae divided by group. Direct Implant group was not included in the graph, since radiotherapy is a relative contraindication for this procedure in our hospital.

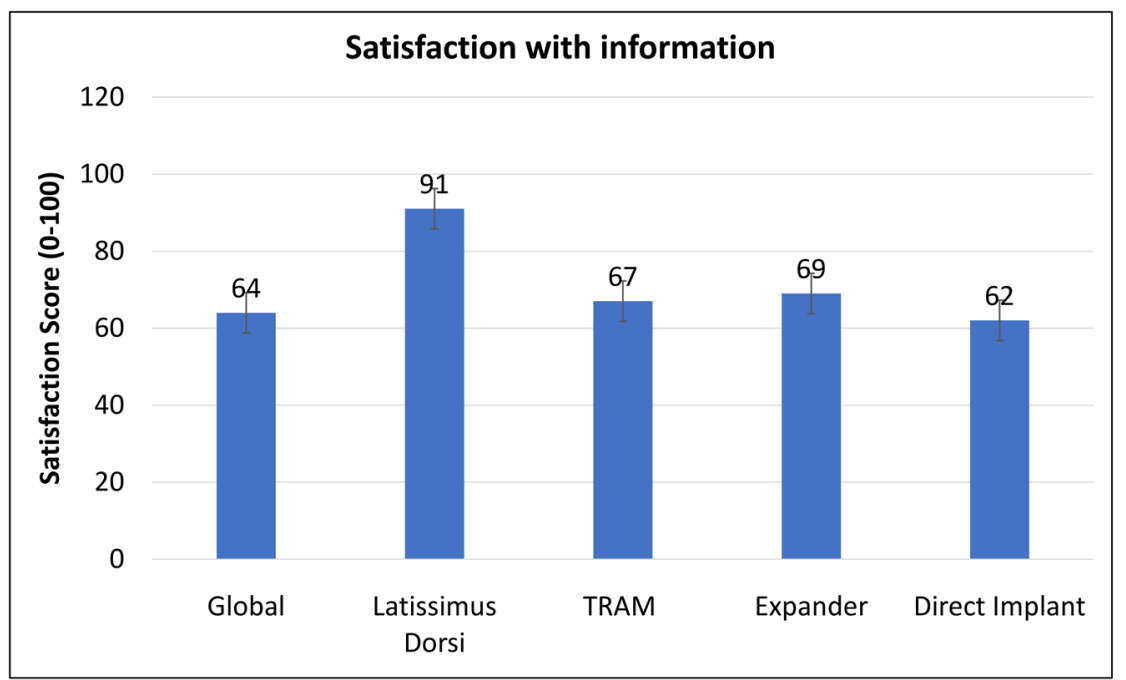

Graphic 6. Satisfaction Score $(+\mathrm{SD})$ regarding information provided preoperatively by the surgeon about the procedure, divided by group.

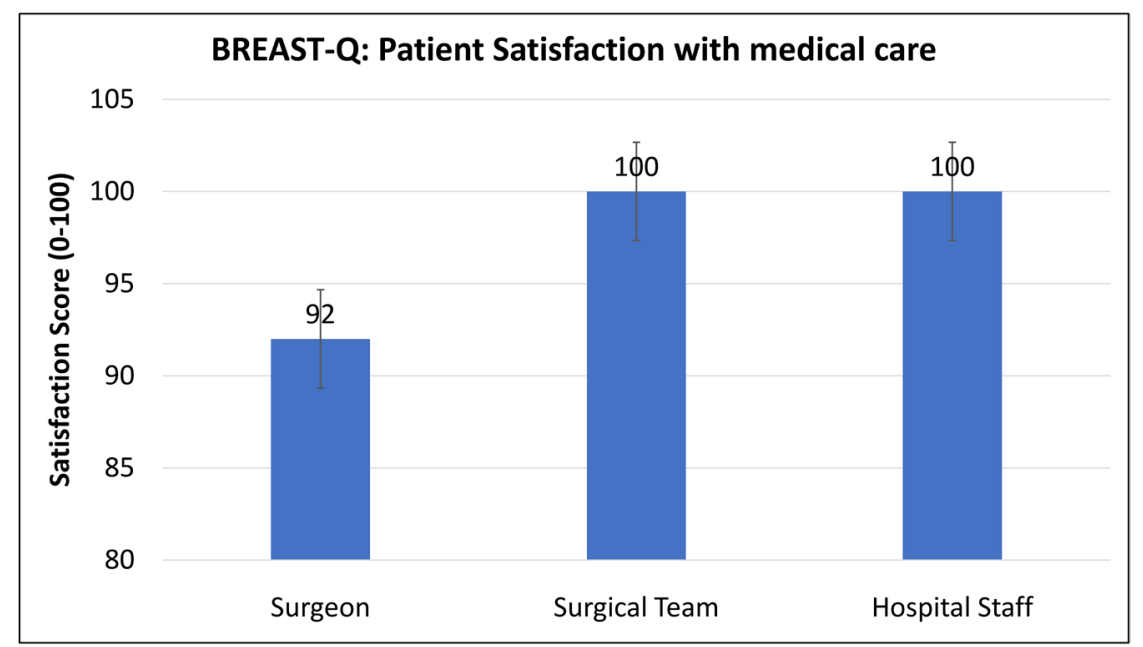

Graphic 7. Global Satisfaction Score (+SD) of medical care divided by provider. 
associated with breast reconstruction [3] [7] [11] [14], but the debate continues over which is the best reconstruction technique.

The PROM-Q are tools that have been gaining popularity in the last few years. They have proven to provide the patient's point of view on their results in an effective and objective way [5] [7] [8] [10] [11], which translate to an improvement in medical care. BREAST-Q in particular is a PROM-Q validated in multiple languages and used in multiple important international studies and has accomplished the task of evaluating patient satisfaction regarding procedures performed in the chest area [8].

In our case, we decided to implement the post reconstruction version of BREAST-Q to evaluate the results in our plastic surgery center, to help us identify areas of opportunity and improve the quality of care to our patients.

In the demographic description of our group, we can observe a homogeneous population that starts the process of reconstruction around the 6th decade of life. The time it took our patients to begin the reconstruction process after mastectomy was in average over 2 years, except for the Expander group. This is due to our oncology and plastic surgery divisions working independently. It is important to consider that this directly affects the quality of care, and directly impacts the patients' quality of life.

Once our patients begin the process of reconstruction, it takes us around 1 year to complete the 4 stages, regardless of the method of reconstruction chosen, which is in accordance with what is written in the literature [15].

Implant based techniques showed a tendency to be safer and was associated with a lower complication index, mostly due to it being a procedure that can be performed in a short time and being less invasive.

A distinctiveness of our study is that autologous tissue breast reconstruction showed a tendency to bring more satisfaction to our patients, despite the duration of the procedure and complication index. These results can be attributed to the use of healthy tissue and aesthetic subunit-based reconstruction, which allowed us to achieve reconstructive goals more often [16] [17]. Satisfaction with the appearance of the chest area proved to be higher than that of the reconstructed breast, which can be related to clothing hiding some imperfections.

Direct implant reconstruction, despite being a simpler technique, was associated with the lowest satisfaction scores amongst our population. This tells us that we must be more careful when selecting patients for this procedure. Once again, the concept of the reconstructive elevator proves valid: the simpler reconstruction will not always yield the best results.

Something that caught our attention was that the incidence of abdominal symptoms amongst the TRAM group was similar to that of the rest of the population, but it was associated with a much higher satisfaction score regarding the appearance of their abdomen.

In our center, one of the main indications for reconstruction with Latissimus Dorsi are radiotherapy sequelae, which was evident in a higher percentage of pa- 
tients that received radiotherapy amongst this group. Nevertheless, the Latissimus Dorsi group was associated with the highest satisfaction and lowest prevalence of radiotherapy sequelae after reconstruction, proving to be an effective strategy to address this issue. In addition, we observed that they are not good candidates for reconstruction with Expander-Implant.

All our patients reported high satisfaction with the care given by the surgeon, surgical team, and hospital staff, yet there are areas of opportunity such as the quality and quantity of information provided to patients.

One of the weaknesses of this study is that we did not perform a preoperatory questionnaire that allowed us to compare the status of these patients prior to surgery, and therefore to effectively measure the changes in this population. It is in our plans to include a preoperatory evaluation with BREAST-Q to continue gather information that will allow us to improve the quality of our care. However, we have observed that it is not practical to apply the full questionnaire in the setting of an outpatient clinic of a public hospital, and due to socio-cultural reasons, it is not feasible to use mobile apps as suggested by Nelson [7]. Therefore, to continue evaluating our results, we have opted to be more selective in the modules to be evaluated.

\section{Conclusions}

Standardized tools such as the BREAST-Q allow us to evaluate medical care and patient perception of results, in a regular and objective manner. Our study, despite not containing a preoperatory evaluation for comparison, shed valuable information due to our patients answering the complete questionnaire, something that less than $50 \%$ of other studies have done [9]. It is also the largest study of this kind in Mexico.

In our center, we observed a tendency towards higher satisfaction index amongst patients who underwent autologous tissue-based reconstruction. Nevertheless, most of the patients that have undergone reconstruction will be satisfied, regardless of the technique used. Therefore, we agree with Alshammari et al. [14], and the statement that every patient is unique, and each method of reconstruction must be carefully evaluated, taking into consideration the patient's situation and opinion.

As doctors and scientists of this era, it is our duty to constantly challenge ourselves and our knowledge. We owe it to those that preceded us to use the new tools and technologies at our disposal to improve patient treatment and our results.

\section{Conflicts of Interest}

The authors declare no conflicts of interest regarding the publication of this paper.

\section{References}

[1] Pan American Health Organization (2018) Cáncer de mama en las Américas. Con- 
sulted on May 20th 2020. Pan American Health Organization, Washington DC. https://www.paho.org/hq/index.php?option=com_docman\&view=download\&categ ory_slug=estadisticas-mapas-4868\&alias $=46503$-epidemiologia-cancer-de-mama-en -las-americas-2018\&Itemid=270\&lang $=$ es

[2] Helms, R.L., O'Hea, E.L. and Corso, M. (2008) Body Image Issues in Women with Breast Cancer. Psychology, Health \& Medicine, 13, 313-325. https://doi.org/10.1080/13548500701405509

[3] Dean, C., Chetty, U. and Forrest, A.P. (1983) Effects of Immediate Breast Reconstruction on Psychosocial Morbidity after Mastectomy. Lancet, 321, 459-462. https://doi.org/10.1016/S0140-6736(83)91452-6

[4] Pinel-Giroux, F.M., El Khoury, M.M., Trop, I., Bernier, C., David, J. and Lalonde, L. (2013) Breast Reconstruction: Review of Surgical Methods and Spectrum of Imaging Findings. RadioGraphics, 33, 435-453. https://doi.org/10.1148/rg.332125108

[5] Cano, S.J., Klassen, A. and Pusic, A.L. (2009) The Science Behind Quality-of-Life Measurement: A Primer for Plastic Surgeons. Plastic and Reconstructive Surgery, 123, 98e-106e. https://doi.org/10.1097/PRS.0b013e31819565c1

[6] Manganiello, A., Hoga, L.A., Reberte, L.M., Miranda, C.M. and Rocha, C.A. (2011). Sexuality and Quality of Life of Breast Cancer Patients Post Mastectomy. European Journal of Oncology Nursing, 15, 167-172. https://doi.org/10.1016/j.ejon.2010.07.008

[7] Nelson, E.C., Eftimovska, E., Lind, C., Hager, A., Wasson, J.H. and Lindblad, S. (2015) Patient Reported Outcome Measures in Practice. BMJ, 350, Article No. g7818. https://doi.org/10.1136/bmj.g7818

[8] Pusic, A.L., Klassen, A.F., Scott, A.M., Klok, J.A., Cordeiro, P.G. and Cano, S.J. (2009) Development of a New Patient-Reported Outcome Measure for Breast Surgery: The BREAST-Q. Plastic and Reconstructive Surgery, 124, 345-353. https://doi.org/10.1097/PRS.0b013e3181aee807

[9] Cohen, W.A., Mundy, L.R., Ballard, T.N., Klassen, A., Cano, S.J., Browne, J. and Pusic, A.L. (2016) The BREAST-Q in Surgical Research: A Review of the Literature 2009-2015. Journal of Plastic, Reconstructive \& Aesthetic Surgery, 69, 149-162. https://doi.org/10.1016/j.bjps.2015.11.013

[10] Lipscomb, J., Gotay, C.C. and Snyder, C.F. (2007) Patient-Reported Outcomes in Cancer: A Review of Recent Research and Policy Initiatives. CA: A Cancer Journal for Clinicians, 57, 278-300. https://doi.org/10.3322/CA.57.5.278

[11] Metcalfe, K.A., Semple, J.L. and Narod, S.A. (2004) Satisfaction with Breast Reconstruction in Women with Bilateral Prophylactic Mastectomy: A Descriptive Study. Plastic and Reconstructive Surgery, 114, 360-366.

https://doi.org/10.1097/01.PRS.0000131877.52740.0E

[12] Kazzazi, F., Haggie, R., Forouhi, P., Kazzazi, N., Wyld, L. and Malata, C.M. (2018). A Comparison of Patient Satisfaction (Using the BREAST-Q Questionnaire) with Bilateral Breast Reconstruction Following Risk-Reducing or Therapeutic Mastectomy. Journal of Plastic, Reconstructive \& Aesthetic Surgery, 71, 1324-1331. https://doi.org/10.1016/j.bjps.2018.06.011

[13] Cano, S.J., Browne, J.P. and Lamping, D.L. (2004) Patient-Based Measures of Outcome in Plastic Surgery: Current Approaches and Future Directions. Journal of Plastic, Reconstructive \& Aesthetic Surgery, 57, 1-11. https://doi.org/10.1016/j.bjps.2003.08.008

[14] Alshammari, S.M., Aldossary, M.Y., Almutairi, K., Almulhim, A., Alkhazmari, G., Alyaqout, M. and Abrar, H. (2019) Patient-Reported Outcomes after Breast Recon- 
structive Surgery: A Prospective Cross-Sectional Study. Annals of Medicine and Surgery, 39, 22-25. https://doi.org/10.1016/j.amsu.2019.02.002

[15] Losken, A., Duggal, C.S., Desai, K.A., McCullough, M.C., Gruszynski, M.A. and Carlson, G.W. (2013) Time to Completion of Nipple Reconstruction: What Factors Are Involved? Annals of Plastic Surgery, 70, 530-532. https://doi.org/10.1097/SAP.0b013e318281ac61

[16] Blondeel, P.N., Hijjawi, J., Depypere, H., Roche, N. and Van Landuyt, K. (2009) Shaping the Breast in Aesthetic and Reconstructive Breast Surgery: An Easy Three-Step Principle. Part II-Breast Reconstruction after Total Mastectomy. Plastic and Reconstructive Surgery, 123, 794-805.

https://doi.org/10.1097/PRS.0b013e318199ef16

[17] Bailey, S.H., Saint-Cyr, M., Oni, G., Maia, M., Andry, D., Shirvani, A., Nguyen, V., Wong, C., Zhang, S., Leitch, A. M., Euhus, D., Rao, R. and Rohrich, R. (2012) Aesthetic Subunit of the Breast: An Analysis of Women's Preference and Clinical Implications. Annals of Plastic Surgery, 68, 240-245.

https://doi.org/10.1097/SAP.0b013e318216b563 